\title{
Von den Balkonen auf die Straßen
}

In der Corona-Pandemie werden Pfleger/innen, Erzieher/innen und Supermarktkassierer/innen als Held/innen gefeiert. Doch nur durch Applaus und Pralinenschachteln lassen sich keine gesellschaftlichen Strukturen verändern - und auch Mieten lassen sich damit nicht bezahlen. Bessere und gerechter verteilte Sorge muss in den patriarchalen Strukturen des Kapitalismus gegen Widerstände erkämpft werden. Von Johanna Ritter, Tobias Kalt und Nilda Inkermann

$\mathbf{S}^{\mathrm{i}}$ ie sind überall im Alltag, und doch oft unsichtbar. Eine Pandemie hat die oft selbstverständlich verrichteten Sorgearbeiten, also umsorgende, vorsorgende und versorgende Tätigkeiten, ins Rampenlicht geholt. Systemrelevant, das waren in dieser Krise nicht mehr die Konzerne und Banken. Systemrelevant, oder besser lebensrelevant, das waren Pfleger/innen, Kassierer/innen, Erntehelfer/innen und die unbezahlte Sorgearbeit zu Hause.

\section{Infrastrukturen der Care-Arbeit}

In dieser Zeit öffnete sich nicht nur das wortwörtliche Fenster für den Applaus von den Balkonen für die Sorgearbeiter/innen, sondern auch das metaphorische für Debatten über relevante Arbeit und Infrastrukturen. Es geht darum, das Prinzip der Sorge - nämlich Leben zu erhalten, Teilhabe zu sichern und Bedürfnisse $\mathrm{zu}$ verwirklichen, ohne andere und die Natur auszubeuten - ins Zentrum einer neuen sozial-ökologischen Wirtschaftsweise zu stellen. Das bedeutet, Infrastrukturen der Care-Arbeit zu verbessern und darüber hinaus, in allen lebensrelevanten Infrastrukturen Sorgebeziehungen zu ermöglichen. So müssten Krankenhäuser viel stärker berücksichtigen, dass Pflege ein soziales Miteinander bedingt und Zeit braucht (siehe Beitrag von Brumbauer et al. in diesem
Heft). Aber auch in anderen Lebensbereichen braucht es sorgeorientierte Infrastrukturen. Die vielen Infektionen in der Fleischindustrie lenken den Blick auf die tier- und menschenunwürdigen Verhältnisse in einem global ausbeuterischen Ernährungssystem. Sorgeorientierte Ernährungsinfrastrukturen umfassen gute Arbeit in der Lebensmittelproduktion und schließen die gesellschaftlichen Naturverhältnisse mit ein. Die vielen Infektionen in der Fleischindustrie machen deutlich, dass es auch in anderen Lebensbereichen sorgeorientierte Infrastrukturen braucht. Nach dem Prinzip der Sorge ist die Natur mehr als stummes Rohstofflager und Mülldeponie. Es erkennt die grundsätzliche Abhängigkeit von ökologischen Systemen an.

Bestehende Infrastrukturen stützen eine profitorientierte Wirtschaftsweise, die im Dienste einzelner Privilegierter steht. Sorgeorientiertes Wirtschaften heißt auch, diese Infrastrukturen abzubauen. Dabei geht es auch um die Verteilung. Unbezahlte und bezahlte Sorgearbeit muss nicht nur aufgewertet, sondern auch gleich verteilt werden. Sie darf nicht weiterhin größtenteils auf den Schultern von Frauen und Migrant/innen lasten.

\section{Viele kleine Schritte}

Für all das standen die Zeichen gut. Für viele kleine Reformschritte gäbe es durchaus große gesellschaftliche Mehr- heiten. Doch warum folgen auf den diskursiven Aufwind keine infrastrukturellen Veränderungen? Vieles hat damit zu tun, dass die Forderung, Sorgearbeit ins Zentrum wirtschaftlicher Aktivitäten zu stellen, die Grundfesten eines patriarchalen, rassistischen und neoliberalen Kapitalismus angreift. Darauf verweist die feministische Ökomomiekritik seit Langem und zeigt auf, dass sorgeorientiertes Wirtschaften kapitalistischen Interessen diametral entgegensteht. Zweifellos braucht es kluge alternative Ideen wie Zeitwohlstand und sozial-ökologische Infrastrukturen. Aber das reicht leider nicht aus. Es braucht nicht nur die guten Ideen, wie es anders geht. Es braucht auch die gesellschaftlichen Kräfte, die diese gegen massiven Widerstand erkämpfen. Kämpfe um eine krisenfeste Gesundheitsversorgung, ökologische Landwirtschaft, Arbeitszeitverkürzung oder ein bedingungsloses Grundeinkommen könnten klassenpolitische, feministische, migrationspolitische und ökologische Anliegen verbinden. Werden diese verknüpft mit einer größeren Erzählung einer wünschenswerten und machbaren alternativen Lebens- und Wirtschaftsweise, ließe sich so gesellschaftliche Gegenmacht für eine sozial-ökologische Transformation aufbauen.

\section{AUTOR/INNEN + KONTAKT}

Johanna Ritter, Tobias Kalt und Nilda Inkermann sind Teil des I. L. A.-Kollektivs („Imperiale Lebensweise und solidarische Alternativen “), in dem sie wissenschaftliche Praxis mit politischen Aktivitäten verknüpfen und sich für Themen globaler Gerechtigkeit, Solidarität und das gute Leben für Alle engagieren

Website: https://ilawerkstatt.org/ Johanna Ritter, Common Future e. V. c/o Thinkfarm, Oberlandstr. 26-35, 12099 Berlin

Tobias Kalt, Universität Hamburg, Mittelweg 117, 20148 Hamburg

Nilda Inkermann, Uni Kassel, Nora Platiel Str. 5, 34109 Kassel
ÖkologischesWirtschaften 4.2020 (35) | DOI 10.14512/OEW350409

(c) 2020 J. Ritter, T. Kalt, N. Inkermann; licensee IÖW and oekom verlag. This is an article distributed under the terms of the Creative Commons Attribution Non-Commercia No Derivates License (http://creativecommons.org/licenses/by-nc-nd/4.o/deed.de) which permits copying and redistributing the material in any medium or format, provided the original work is properly cited, it is not used for commercial purposes and it is not remixed, transformed or built upon. The access to the digital version of this article is reserved to subscribers of ÖkologischesWirtschaften until two years after the date of publication; after two years it is available to all readers. 